\title{
High-level Emergency Care Centers and Hospitals with Both Medical/Surgical and Psychiatric Inpatient Beds are associated with a lower rate of difficulty in hospital acceptance and shorter prehospital transfer time for patients seeking care after self-harm behavior
}

Takuyo Chiba ( $\nabla$ c.takuyo@gmail.com )

International University of Health and Welfare, Narita Hospital

Takaku Reo

Institute for Health Economics and Policy

Erina Ito

School of International and Public Policy, Hitotsubashi University

Hidetaka Tamune

The University of Tokyo

Marisa Rivera

Chiba University Hospital

Shunya Ikeda

International University of Health and Welfare

Takashi Shiga

International University of Health and Welfare, Narita Hospital

Research Article

Keywords: age, gender, the population density, emergency care, medical/surgical and psychiatric inpatient

Posted Date: July 9th, 2021

DOl: https://doi.org/10.21203/rs.3.rs-690906/v1

License: (c) (i) This work is licensed under a Creative Commons Attribution 4.0 International License.

Read Full License 


\section{Abstract}

Ambulance diversion has a significant impact on patient care outcomes. Self-harm behavior in particular is associated with difficulty in hospital acceptance. This is a retrospective observational study using the Japanese ambulance dispatch data in 2015. This study aims to determine if high-level emergency care centers and hospitals with both medical/surgical and psychiatric inpatient beds are associated with a decreased rate of difficulty in hospital acceptance and shorter prehospital transfer times for patients seeking medical care after self-harm behavior using 2015 Japanese ambulance dispatch database. Multivariable analyses were performed for difficulty in hospital acceptance and prehospital transfer time. Covariates such as age, gender, the population density, time, and severity, were included in the analysis, in addition to the presence of high-level emergency care centers and hospitals with both medical/surgical and psychiatric inpatient beds in the city. There was an association between decreased difficulty in hospital acceptance and the presence of high-level emergency care centers (Odds ratio $0.62,95 \%$ Confidence Interval 0.55 to $0.70, \mathrm{P}<0.01)$ and hospitals with both medical/surgical and psychiatric inpatient beds $(\mathrm{OR}=0.49,95 \% \mathrm{Cl}, 0.37$ to $0.65, \mathrm{P}<0.01)$. There was significant reduction in prehospital transfer time in the city with high-level emergency care centers $(4.21 \mathrm{~min}, 95 \% \mathrm{Cl} 3.53$ to $4.89, \mathrm{P}<0.01)$ and hospitals with medical/surgical and psychiatric inpatient beds $(3.46 \mathrm{~min}, 95 \% \mathrm{Cl} 2.15$ to $4.77, \mathrm{P}<$ 0.01). High-level emergency care centers and hospitals with both medical/surgical and psychiatric inpatient beds were associated with significant decrease in difficulty in hospital acceptance and shorter prehospital transfer time. (The graphical abstract is shown in Fig. 1.)

\section{Introduction}

For the last thirty years, ambulance diversion has been a major issue since it was adopted as a measure to mitigate emergency department overcrowding ${ }^{1,2}$. Ambulance diversion was associated with increased mortality in patients with acute myocardial infarction and trauma ${ }^{3-5}$. Decreasing ambulance diversion and the time of emergency medical service transfer could have a significant clinical impact on patients' outcomes. The rate of emergency department visits related to suicidal attempt or suicidal ideation has been increasing, and approximately 1.5 million emergency department visits are due to suicidal attempt and suicidal ideation in $2017^{6}$. Given the steady increase of emergency department visits for patients with psychiatric problems, the effective way to minimize ambulance diversion is needed. A previous study reported that self-induced drug abuse/gas poisoning and self-induced trauma were associated with increased difficulty in hospital acceptance at the scene ${ }^{7}$. This is likely due to concerns for possible underlying psychiatric illness, which pose clinical challenges to some hospitals that do not have psychiatric resources. Despite the rising need for hospitals that can manage both physical and psychiatric issues, the number of hospitals with both medical/surgical and psychiatric inpatient beds(MSPHs) recently have been decreasing in Japan ${ }^{8,9}$.

Currently, Japan does not have any nation-wide regulations to restrict ambulance diversion. Although reducing prehospital transfer time is an important clinical issue, there is insufficient data about factors 
associated with the increased ambulance diversion rate in patients seeking care after self-harm behavior.

We presumed that the lack of mental health resources is a major obstacle for hospitals to accept patients with self-harm behavior. In this study, we aim to determine if the presence of tertiary care emergency departments with psychiatric services or hospitals with both medical/surgical and psychiatric inpatient beds is associated with decreased rate of difficulty in hospital acceptance at the scene and shorter prehospital transfer time for patients with self-harm behavior.

\section{Methods}

Emergency medicine system in Japan

The Emergency Medicine System (EMS) in Japan is run by local governments and available to everyone who needs emergency transport to a hospital without any direct payment. An ambulance responds to a 119 call and picks up a patient at the scene. After evaluating the patient, the EMS must find an accepting hospital that can provide optimal care in the area. With the exception of a few areas, such as Tokyo, there are no systematic regulations that prevent ambulance diversion and each hospital can decide to accept the patient based on capacity and capability. Sometimes multiple phone calls are required to find an accepting hospital.

Currently, there are 265 level three emergency care centers (EC3s) (designated critical care hospitals) in Japan to accept severely ill or injured patients due to stroke, acute myocardial infarction, cardiopulmonary arrest, trauma, etc. A hospital must meet certain criteria to be appointed as an EC3. One requirement is the availability of psychiatric care on request, however this is not essential and not all EP3s have on-call psychiatrists.

MSPHs have medical and surgical specialists for physical disease as well as psychiatrists for psychiatric issues. However, medical resources at these facilities may be limited compared to EC3s.

Study design, population, and setting

This is a retrospective observational study using a Japanese national database of nation-wide ambulance dispatches in 2015. The database does not include ambulance transfers in the Tokyo area. We collected cases of ambulance transfers due to self-harm behavior. The Japanese Population Census performed in 2015 was used to collect data regarding municipal population. Static/Dynamic Surveys of Medical Institutions and Hospitals conducted by the Ministry of Health, Labor and Welfare in 2015 was referenced for the number of inpatient beds. The information about hospitals with medical and psychiatric inpatient beds was provided by Japanese Society of General Hospital Psychiatry. This research was approved and the informed consent was waived by the ethics committee at the International University of Health and Welfare because the study data did not contain identifiable protected health information. (Institutional Review Board Approval Number 13-B-375) The study was 
carried out in accordance with the Declaration of Helsinki and Ethical Guidelines for Medical and Health Research Involving Human Subjects published by Ministry of Health, Labor and Welfare in Japan.

Data collection and quality control

A previous study using the same database has already described how the information were collected ${ }^{10}$. Data was collected uniformly using specific data collection forms which include age, gender, location of call, time of the day, chronological factors such as time of the day or day of the week, time of ambulance request, time of arrival at the scene, time of arrival at the accepting hospital, diagnosis, and severity. The forms were completed by EMS personnel and confirmed by peer EMS personnel to ensure data accuracy. The treating physicians provided information regarding the patient's diagnosis and severity in the emergency department. The definition of a "severe condition" is intensive care unit admission and a "moderate condition" is defined by hospital admission. Daytime is from 800 to 1700 . A designated supervising officer at each fire station assures the completeness of transport data. The data was gathered from EMS administrative records collected by the fire department and not connected to a patient's medical chart.

\section{Endpoints}

The primary outcome is the rate of cases with difficulty in hospital acceptance as defined by four or more phone calls before deciding the facility to transfer the patient. This definition is used by the Ministry of Internal Affairs and Communications in White Paper on Fire Service. The secondary outcome is prehospital transfer time defined by the time between the arrival at the scene and the hospital arrival.

\section{Statistical analysis}

Univariate analysis was performed between the groups of patients with and without difficulty in hospital acceptance regarding baseline demographics using T-tests for continuous variables and chi-square tests for binominal variables. Similarly, univariate analysis was performed for prehospital transfer times regarding some baseline characteristics. Then, a multivariable logistic regression model was applied for the assessment of the difficulty in hospital acceptance. A multivariable linear regression model was applied to analyze factors associated with the prehospital transfer time. Factors included in the analysis were age, gender (male or female), rate of elderly (65 years old or older), the population density, time of the day (day or night), day of the week (weekday or weekend/holiday), severity (mild, moderate, severe, death), location (public space, workplace, street, or other), the number of inpatient beds per population in the city, the third digit of the Municipal Code assigned by Ministry of Internal Affairs and Communications which indicated the size and function of the city, the time between the initial ambulance request call and the arrival at the scene, number of emergency care centers that are not EC3s, presence of hospitals with only psychiatric service in the city, the presence of EC3s, and MSPHs. Interaction terms were adopted for the presence of EC3s and MSPHs. Stata version 14 (College Station, TX) was utilized for statistical analysis. All tests were two-tailed, and P-value less than 0.05 was considered statistically significant. 


\section{Results}

The number of total ambulance dispatches was 4,805,224, and ambulance transfer due to self-harm behavior was 34,699. After excluding 1,850 transfers with missing data, 32,849 cases were enrolled in the study. (Fig. 2.) Basic demographic information is shown in Table 1. The mean age was 45.0 years old and females account for $61.6 \%$ of transfers. Table 2 shows overall prehospital transfer time and prehospital transfer time in subgroups. Cases with difficulty in hospital acceptance were $8.4 \%(2,767$ transfers) and the mean prehospital transfer time was 37.1 minutes. 
Table 1

Baseline characteristics of the patients transferred for self-harm behavior. Univariate analyses were performed with t-test and chi-square test for continuous valuables and binominal valuables, respectively. Factors showed significant difference were marked with asterisks. $\star \star \star ~ P<0.01 * P<0.1$

\section{Baseline Characteristics}

\begin{tabular}{|c|c|c|c|c|}
\hline & $\begin{array}{l}\text { All transfer }(\mathrm{N} \\
=32,849)\end{array}$ & $\begin{array}{l}\text { Phone calls } \geqq 4 \\
(N=2,767)\end{array}$ & $\begin{array}{l}\text { Phone calls }<4 \\
(\mathrm{~N}=30,082)\end{array}$ & \\
\hline Age(SD) & $45.0(19.1)$ & 41.5(17.1) & $45.4(19.1)$ & $\star \star \star \star ~$ \\
\hline Female sex(\%) & $20,283(61.6)$ & $1,912(69.1)$ & $18,319(60.9)$ & *** \\
\hline Elderly rate (SD) & $26.6(4.0)$ & $26.2(3.4)$ & $26.6(4.1)$ & *** \\
\hline Population density (SD) $†$ & $305.6(339.6)$ & $459.0(427.8)$ & 291.5(326.7) & *** \\
\hline Hospital beds per capita (SD)‡ & $132.3(59.5)$ & $120.4(46.6)$ & $133.4(60.5)$ & *** \\
\hline Night-time transfer (\%) & $21,181(64.5)$ & $2,027(73.3)$ & $19,154(63.7)$ & *** \\
\hline Weekend/Holiday transfer (\%) & $9,692(29.5)$ & $883(31.9)$ & $8,809(29.3)$ & *** \\
\hline \multicolumn{5}{|l|}{ Severity (\%) } \\
\hline Mild & $11,400(34.7)$ & $1,150(41.6)$ & $10,250(34.1)$ & *** \\
\hline Moderate & $12,408(37.8)$ & $1,288(46.6)$ & $11,120(37.0)$ & \\
\hline Severe & $4,893(14.9)$ & 192(6.9) & $4,701(15.6)$ & \\
\hline Death & $4,148(12.6)$ & $137(5.0)$ & $4,011(13.3)$ & \\
\hline \multicolumn{5}{|l|}{ Location(\%) } \\
\hline Home & $26,463(80.6)$ & $2,308(83.4)$ & $24,155(80.3)$ & $\star \star \star ~$ \\
\hline Public space & $2,743(8.4)$ & 194(7.0) & $2,549(8.5)$ & \\
\hline Workplace & $342(1.0)$ & $19(0.7)$ & $323(1.1)$ & \\
\hline Road & $1,267(3.9)$ & 109(3.9) & $1,158(3.9)$ & \\
\hline Other & $2,034(6.2)$ & $137(5.0)$ & $1,897(6.3)$ & \\
\hline $\begin{array}{l}\text { Level three emergency care centers } \\
(\%)\end{array}$ & $20,387(62.1)$ & $1,648(59.6)$ & $18,739(62.3)$ & *** \\
\hline $\begin{array}{l}\text { Hospitals with Medical/Surgical and } \\
\text { Psychiatric wards (\%) }\end{array}$ & $14,823(45.1)$ & $1,243(44.9)$ & $13,580(45.1)$ & \\
\hline $\begin{array}{l}\text { Hospitals with only psychiatric } \\
\text { service(\%) }\end{array}$ & $26,992(82.2)$ & $2,240(81.0)$ & $24,752(82.3)$ & * \\
\hline Emergency care center(\%) & 32292(98.3) & $2,740(99.1)$ & $29,582(98.2)$ & \\
\hline
\end{tabular}




\section{Baseline Characteristics}

$\star * * P<0.01$

** $\mathrm{P}<0.05$

$* P<0.1$

† Population density unit = population/squire kilometer

$\ddagger$ Hospital beds per capita unit $=$ hospital beds $/ 1000$ population 
Table 2

Prehospital transfer time in subgroups. Analyses with t-test were performed and factors with significant difference were marked with asterisks. ** $\mathrm{P}<0.01$

\section{Prehospital Transfer Time (min)}

\section{All transfer $(\mathrm{N})$}

37.1(22.8)

$\operatorname{Sex}(n)$

Male $35.3(22.7) \quad * * *$

Female $38.2(22.8)$

Time ( $n)$

Night-time

$38.1(23.2) \quad * \star \star$

Day-time

Severity ( $n$

Mild

$\star \star \star ~$

Moderate

$41.0(25.0)$

Severe

$31.9(20.8)$

Death

25.5(12.7)

Level three emergency care centers (n)

Presence

$36.2(21.9) \quad * \star \star$

Absence

$38.5(24.1)$

Hospitals with Medical/Surgical and Psychiatric wards (n)

Presence

$36.7(22.5) \quad * \star \star$

Absence

$37.5(23.0)$

Hospitals with only psychiatric service (n)

Presence

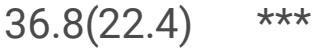

Absence

$38.7(24.4)$

$\star \star \star \mathrm{P}<0.01$

$\star * \mathrm{P}<0.05$

$* P<0.1$

Table 3 is the result of multivariable logistic regression analysis for factors associated with difficulty in hospital acceptance. Table 4 is the result of multivariable linear regression analysis for factors 
associated with prehospital transfer time. EC3s (OR $0.63,95 \% \mathrm{Cl} 0.55$ to $0.71, \mathrm{P}<0.01$ ), and MSPHs (OR $0.50,95 \% \mathrm{Cl} 0.38$ to $0.66, \mathrm{P}<0.01$ ) were associated with decreased difficulty in hospital acceptance after adjusting other factors with multivariable regression model. In terms of prehospital transfer time, EC3s $(-4.21,95 \% \mathrm{Cl}-3.53$ to $-4.89, \mathrm{P}<0.01)$ and $\mathrm{MSPHs}(-3.46,95 \% \mathrm{Cl}-2.15$ to $-4.77, \mathrm{P}<0.01)$ were associated with shorter prehospital transfer time. Focusing on the interaction terms between the presence of EC3s and MSPHs, the effect of EC3s (OR 0.92) and MSPHs (OR 0.73) on the difficulty in hospital acceptance were decreased when the other was present in the same city. Similarly, the presence of EC3s $(-1.10 \mathrm{~min})$ and MSPHs (-0.35 $\mathrm{min})$ were associated with a smaller decrease of prehospital transfer time when the other is located in the same city. 
Table 3

The result of multivariable logistic regression analysis for difficulty in hospital acceptance. $95 \% \mathrm{Cl}$ in parentheses. *** $p<0.01, * \star p<0.05, * p<0.1$

\section{Multivariable Logistic Regression Analysis for Difficulty in Hospital}

Acceptance

\begin{tabular}{|c|c|c|c|c|c|}
\hline & All & $\begin{array}{l}\text { Night- } \\
\text { time }\end{array}$ & Day-time & $\begin{array}{l}\text { Weekend/ } \\
\text { Holiday }\end{array}$ & Weekday \\
\hline \multirow[t]{2}{*}{ EC3s } & $0.63^{\star \star *}$ & $0.63^{\star \star \star}$ & $0.61^{\star \star \star}$ & $0.77^{\star \star}$ & $0.57 \star \star \star$ \\
\hline & $\begin{array}{l}(0.55- \\
0.71)\end{array}$ & $\begin{array}{l}(0.55- \\
0.73)\end{array}$ & $\begin{array}{l}(0.49- \\
0.77)\end{array}$ & $\begin{array}{l}(0.62- \\
0.95)\end{array}$ & $\begin{array}{l}(0.49- \\
0.66)\end{array}$ \\
\hline \multirow[t]{2}{*}{ MSPHs } & $0.50 * \star \star *$ & $0.51^{\star \star \star}$ & $0.48^{\star * \star}$ & $0.57^{\star \star}$ & $0.47^{\star \star \star}$ \\
\hline & $\begin{array}{l}(0.38- \\
0.66)\end{array}$ & $\begin{array}{l}(0.37- \\
0.70)\end{array}$ & $\begin{array}{l}(0.28- \\
0.83)\end{array}$ & $\begin{array}{l}(0.34- \\
0.94)\end{array}$ & $\begin{array}{l}(0.34- \\
0.65)\end{array}$ \\
\hline \multirow{2}{*}{$\begin{array}{l}\text { Interaction terms (MSPH and } \\
\text { EC3) }\end{array}$} & $1.47^{\star \star}$ & $1.40 *$ & 1.62 & 1.23 & $1.59 \star \star$ \\
\hline & $\begin{array}{l}(1.07- \\
2.01)\end{array}$ & $\begin{array}{l}(0.97- \\
2.03)\end{array}$ & $\begin{array}{l}(0.87- \\
3.01)\end{array}$ & $\begin{array}{l}(0.69- \\
2.20)\end{array}$ & $\begin{array}{l}(1.09- \\
2.33)\end{array}$ \\
\hline \multirow[t]{2}{*}{ Psychiatry only hospitals } & 0.96 & 0.95 & 0.99 & 1.02 & 0.93 \\
\hline & $\begin{array}{l}(0.85- \\
1.08)\end{array}$ & $\begin{array}{l}(0.82- \\
1.09)\end{array}$ & $\begin{array}{l}(0.80- \\
1.23)\end{array}$ & $\begin{array}{l}(0.82- \\
1.28)\end{array}$ & $\begin{array}{l}(0.81- \\
1.07)\end{array}$ \\
\hline \multirow{2}{*}{$\begin{array}{l}\text { Time from dispatch to arrival at } \\
\text { the scene }\end{array}$} & $1.02^{\star \star \star}$ & $1.02^{\star \star}$ & $1.02^{\star \star}$ & 1.01 & $1.02^{\star \star \star}$ \\
\hline & $\begin{array}{l}(1.01- \\
1.02)\end{array}$ & $\begin{array}{l}(1.00- \\
1.03)\end{array}$ & $\begin{array}{l}(1.00- \\
1.03)\end{array}$ & $\begin{array}{l}(0.99- \\
1.03)\end{array}$ & $\begin{array}{l}(1.01- \\
1.03)\end{array}$ \\
\hline \multirow[t]{2}{*}{ Emergency care center } & $1.02^{\star \star \star}$ & 1.01 ** & $1.02^{\star \star *}$ & $1.01^{\star \star \star}$ & $1.02^{\star \star \star}$ \\
\hline & $\begin{array}{l}(1.01- \\
1.02)\end{array}$ & $\begin{array}{l}(1.01- \\
1.02)\end{array}$ & $\begin{array}{l}(1.01- \\
1.02)\end{array}$ & $\begin{array}{l}(1.01- \\
1.02)\end{array}$ & $\begin{array}{l}(1.01- \\
1.02)\end{array}$ \\
\hline \multirow[t]{2}{*}{ Age } & $1.00^{\star \star \star}$ & $1.00^{\star \star \star}$ & 01.00 ** & $0.99 \star \star \star$ & $1.00 \star \star$ \\
\hline & $\begin{array}{l}(0.99- \\
1.00)\end{array}$ & $\begin{array}{l}(0.99- \\
1.00)\end{array}$ & $\begin{array}{l}(0.99- \\
1.00)\end{array}$ & $\begin{array}{l}(0.99- \\
1.00)\end{array}$ & $\begin{array}{l}(0.99- \\
1.00)\end{array}$ \\
\hline \multirow[t]{2}{*}{ Female sex } & $1.11^{\star \star}$ & $1.14 \star \star$ & 1.07 & $1.18^{\star \star}$ & 1.09 \\
\hline & $\begin{array}{l}(1.02- \\
1.22)\end{array}$ & $\begin{array}{l}(1.02- \\
1.26)\end{array}$ & $\begin{array}{l}(0.91- \\
1.27)\end{array}$ & $\begin{array}{l}(1.01- \\
1.38)\end{array}$ & $\begin{array}{l}(0.98- \\
1.21)\end{array}$ \\
\hline \multirow[t]{2}{*}{ Elderly rate } & $1.02^{\star \star \star}$ & $1.02^{\star \star \star}$ & 1.01 & 1.02 & $1.02^{\star \star \star}$ \\
\hline & $\begin{array}{l}(1.01- \\
1.03)\end{array}$ & $\begin{array}{l}(1.01- \\
1.04)\end{array}$ & $\begin{array}{l}(0.99- \\
1.03)\end{array}$ & $\begin{array}{l}(1.00- \\
1.04)\end{array}$ & $\begin{array}{l}(1.01- \\
1.03)\end{array}$ \\
\hline
\end{tabular}




\begin{tabular}{|c|c|c|c|c|c|}
\hline \multicolumn{6}{|c|}{$\begin{array}{l}\text { Multivariable Logistic Regression Analysis for Difficulty in Hospital } \\
\text { Acceptance }\end{array}$} \\
\hline \multirow[t]{2}{*}{ Population density } & $1.08^{\star \star \star}$ & $1.10^{\star \star \star}$ & 1.02 & $1.09 * * *$ & $1.07 * \star \star$ \\
\hline & $\begin{array}{l}(1.06- \\
1.10)\end{array}$ & $\begin{array}{l}(1.08- \\
1.12)\end{array}$ & $\begin{array}{l}(0.99- \\
1.05)\end{array}$ & $\begin{array}{l}(1.05- \\
1.12)\end{array}$ & $\begin{array}{l}(1.05- \\
1.09)\end{array}$ \\
\hline \multirow[t]{2}{*}{ Night time } & $1.45^{\star \star \star}$ & & & $1.34^{\star \star \star}$ & $1.50 * \star \star$ \\
\hline & $\begin{array}{l}(1.32- \\
1.58)\end{array}$ & & & $\begin{array}{l}(1.14- \\
1.57)\end{array}$ & $\begin{array}{l}(1.34- \\
1.67)\end{array}$ \\
\hline \multirow[t]{2}{*}{ Weekend/Holiday } & $1.13^{\star \star \star}$ & $1.10 *$ & $1.22^{\star \star}$ & & \\
\hline & $\begin{array}{l}(1.04- \\
1.23)\end{array}$ & $\begin{array}{l}(0.99- \\
1.22)\end{array}$ & $\begin{array}{l}(1.04- \\
1.43)\end{array}$ & & \\
\hline \multicolumn{6}{|l|}{ Severity $($ baseline $=$ mild $)$} \\
\hline \multirow[t]{2}{*}{ Moderate } & $1.12^{\star \star \star}$ & 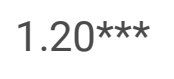 & 0.94 & 1.09 & $1.13^{\star \star}$ \\
\hline & $\begin{array}{l}(1.03- \\
1.22)\end{array}$ & $\begin{array}{l}(1.08- \\
1.32)\end{array}$ & $\begin{array}{l}(0.80- \\
1.11)\end{array}$ & $\begin{array}{l}(0.934- \\
1.272)\end{array}$ & $\begin{array}{l}(1.02- \\
1.26)\end{array}$ \\
\hline \multirow[t]{2}{*}{ Severe } & $0.46^{\star \star \star}$ & $0.48^{\star \star \star}$ & 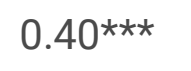 & $0.53^{\star \star \star}$ & $0.42^{\star \star \star}$ \\
\hline & $\begin{array}{l}(0.39- \\
0.54)\end{array}$ & $\begin{array}{l}(0.39- \\
0.58)\end{array}$ & $\begin{array}{l}(0.30-- \\
0.53)\end{array}$ & $\begin{array}{l}(0.40- \\
0.70)\end{array}$ & $\begin{array}{l}(0.35- \\
0.52)\end{array}$ \\
\hline \multirow[t]{2}{*}{ Death } & $0.37 \star \star \star$ & $0.43^{\star \star \star}$ & $0.26 * \star \star$ & $0.47^{\star \star \star}$ & $0.33^{\star \star \star}$ \\
\hline & $\begin{array}{l}(0.31- \\
0.45)\end{array}$ & $\begin{array}{l}(0.34- \\
0.54)\end{array}$ & $\begin{array}{l}(0.19- \\
0.38)\end{array}$ & $\begin{array}{l}(0.34- \\
0.65)\end{array}$ & $\begin{array}{l}(0.26- \\
0.42)\end{array}$ \\
\hline \multicolumn{6}{|l|}{ Place (baseline = home) } \\
\hline \multirow[t]{2}{*}{ Public place } & $0.83^{\star \star}$ & 0.88 & $0.67 * \star$ & 0.83 & $0.82 \star \star$ \\
\hline & $\begin{array}{l}(0.707- \\
0.964)\end{array}$ & $\begin{array}{l}(0.74- \\
1.06)\end{array}$ & $\begin{array}{l}(0.49- \\
0.92)\end{array}$ & $\begin{array}{l}(0.62- \\
1.10)\end{array}$ & $\begin{array}{l}(0.68- \\
0.99)\end{array}$ \\
\hline \multirow[t]{2}{*}{ Work place } & 0.93 & 1.01 & 0.8 & 0.66 & 1.04 \\
\hline & $\begin{array}{l}(0.58- \\
1.51)\end{array}$ & $\begin{array}{l}(0.55- \\
1.86)\end{array}$ & $\begin{array}{l}(0.36- \\
1.76)\end{array}$ & $\begin{array}{l}(0.24- \\
1.78)\end{array}$ & $\begin{array}{l}(0.60- \\
1.81)\end{array}$ \\
\hline \multirow[t]{2}{*}{ Road } & 0.94 & 0.92 & 1.02 & 1.01 & 0.92 \\
\hline & $\begin{array}{l}(0.77- \\
1.16)\end{array}$ & $\begin{array}{l}(0.72- \\
1.16)\end{array}$ & $\begin{array}{l}(0.67- \\
1.55)\end{array}$ & $\begin{array}{l}(0.71- \\
1.43)\end{array}$ & $\begin{array}{l}(0.71- \\
1.18)\end{array}$ \\
\hline \multirow[t]{2}{*}{ Other } & 0.86 & 0.95 & $0.66^{\star \star}$ & 0.88 & 0.86 \\
\hline & $\begin{array}{l}(0.72- \\
1.04)\end{array}$ & $\begin{array}{l}(0.76- \\
1.17)\end{array}$ & $\begin{array}{l}(0.46- \\
0.97)\end{array}$ & $\begin{array}{l}(0.64- \\
1.21)\end{array}$ & $\begin{array}{l}(0.68- \\
1.07)\end{array}$ \\
\hline Hospital beds per capita & $1.00 * \star \star$ & $1.00 * \star$ & $01.00 * * *$ & $1.00 *$ & $1.00 \star \star \star$ \\
\hline
\end{tabular}


Multivariable Logistic Regression Analysis for Difficulty in Hospital

Acceptance

\begin{tabular}{llllll} 
& $(1.00-$ & $(1.00-$ & $(1.00-$ & $(1.00-$ & $(1.00-$ \\
& $1.00)$ & $1.00)$ & $1.00)$ & $1.00)$ & $1.00)$ \\
Observations & 32,849 & 21,181 & 11,668 & 9,692 & 23,157 \\
$95 \% \mathrm{Cl}$ in parentheses & & & & & \\
*** $\mathrm{p}<0.01, * * \mathrm{p}<0.05, * \mathrm{p}<0.1$ & & & & & \\
\hline
\end{tabular}


Table 4

The result of multivariable linear regression analysis for prehospital transfer time. $95 \% \mathrm{Cl}$ in parentheses. $\star \star \star ~ p<0.01, \star \star p<0.05, * p<0.1$

Table 4. Multivariable Linear Regression Analysis for Prehospital Transfer Times

\begin{tabular}{|c|c|c|c|c|c|}
\hline & All & Night-time & Day-time & $\begin{array}{l}\text { Weekend/ } \\
\text { Holiday }\end{array}$ & Weekday \\
\hline \multirow[t]{2}{*}{ EC3s } & 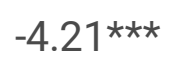 & $-4.38 * \star \star$ & $-3.931 * * *$ & $-3.83^{\star \star \star}$ & $-4.37 * \star \star$ \\
\hline & $\begin{array}{l}(-4.89- \\
-3.53)\end{array}$ & $\begin{array}{l}(-5.25- \\
-3.50)\end{array}$ & $\begin{array}{l}(-5.01- \\
-2.86)\end{array}$ & $\begin{array}{l}(-5.10- \\
-2.56)\end{array}$ & $\begin{array}{l}(-5.18- \\
-3.57)\end{array}$ \\
\hline \multirow[t]{2}{*}{ MSPHs } & $-3.46 * \star \star$ & 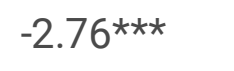 & $-4.63^{\star \star \star}$ & $-3.20 * \star \star$ & 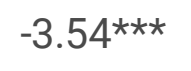 \\
\hline & $\begin{array}{l}(-4.77- \\
-2.15)\end{array}$ & $\begin{array}{l}(-4.54- \\
-0.97)\end{array}$ & $\begin{array}{l}(-6.39- \\
-2.88)\end{array}$ & $\begin{array}{l}(-5.58- \\
-0.82)\end{array}$ & $\begin{array}{l}(-5.12- \\
-1.97)\end{array}$ \\
\hline \multirow{2}{*}{$\begin{array}{l}\text { Interaction terms (MSPH and } \\
\text { EC3) }\end{array}$} & $3.11^{\star \star \star}$ & $2.18^{\star \star}$ & $4.74^{\star \star \star}$ & 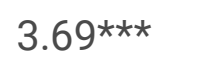 & 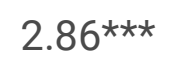 \\
\hline & $\begin{array}{l}(1.66- \\
4.57)\end{array}$ & $\begin{array}{l}(0.22- \\
4.13)\end{array}$ & $\begin{array}{l}(2.68- \\
6.81)\end{array}$ & $\begin{array}{l}(0.98- \\
6.40)\end{array}$ & $\begin{array}{l}(1.12- \\
4.59)\end{array}$ \\
\hline \multirow[t]{2}{*}{ Psychiatry only hospitals } & 0.21 & 0.41 & -0.12 & -0.38 & 0.46 \\
\hline & $\begin{array}{l}(-0.53- \\
0.94)\end{array}$ & $\begin{array}{l}(-0.52- \\
1.35)\end{array}$ & $\begin{array}{l}(-1.32- \\
1.08)\end{array}$ & $\begin{array}{l}(-1.78- \\
1.03)\end{array}$ & $\begin{array}{l}(-0.40- \\
1.32)\end{array}$ \\
\hline \multirow{2}{*}{$\begin{array}{l}\text { Time from dispatch to arrival } \\
\text { at the scene }\end{array}$} & $0.73^{\star \star \star}$ & $0.78^{\star \star *}$ & $0.66^{\star \star \star}$ & $0.74 * \star \star$ & $0.73^{\star \star \star}$ \\
\hline & $\begin{array}{l}(0.65- \\
0.82)\end{array}$ & $\begin{array}{l}(0.67- \\
0.89)\end{array}$ & $\begin{array}{l}(0.54- \\
0.78)\end{array}$ & $\begin{array}{l}(0.57- \\
0.90)\end{array}$ & $\begin{array}{l}(0.64- \\
0.83)\end{array}$ \\
\hline \multirow[t]{2}{*}{ Emergency care center } & $0.06^{\star \star \star}$ & $0.06^{* * *}$ & $0.05^{\star \star}$ & $0.06^{\star \star}$ & $0.06^{\star \star \star}$ \\
\hline & $\begin{array}{l}(0.04- \\
0.08)\end{array}$ & $\begin{array}{l}(0.04- \\
0.09)\end{array}$ & $\begin{array}{l}(0.01- \\
0.08)\end{array}$ & $\begin{array}{l}(0.01- \\
0.10)\end{array}$ & $\begin{array}{l}(0.03- \\
0.09)\end{array}$ \\
\hline \multirow[t]{2}{*}{ Age } & $-0.02^{\star \star \star}$ & $-0.02^{\star}$ & $-0.03 * \star \star$ & -0.02 & $-0.02 * \star \star$ \\
\hline & $\begin{array}{l}(-0.03- \\
-0.01)\end{array}$ & $\begin{array}{l}(-0.03- \\
0.00)\end{array}$ & $\begin{array}{l}(-0.05- \\
-0.01)\end{array}$ & $\begin{array}{l}(-0.04- \\
0.01)\end{array}$ & $\begin{array}{l}(-0.04- \\
-0.01)\end{array}$ \\
\hline \multirow[t]{2}{*}{ Female sex } & 0.20 & 0.03 & 0.47 & 0.66 & 0.01 \\
\hline & $\begin{array}{l}(-0.31- \\
0.71)\end{array}$ & $\begin{array}{l}(-0.63- \\
0.69)\end{array}$ & $\begin{array}{l}(-0.32- \\
1.26)\end{array}$ & $\begin{array}{l}(-0.27- \\
1.58)\end{array}$ & $\begin{array}{l}(-0.60- \\
0.62)\end{array}$ \\
\hline \multirow[t]{2}{*}{ Elderly rate } & $0.24^{\star \star \star}$ & $0.26 * \star \star$ & $0.21 * \star \star$ & $0.24 * \star \star$ & $0.25^{\star \star \star}$ \\
\hline & $\begin{array}{l}(0.17- \\
0.32)\end{array}$ & $\begin{array}{l}(0.17- \\
0.36)\end{array}$ & $\begin{array}{l}(0.09- \\
0.32)\end{array}$ & $\begin{array}{l}(0.10- \\
0.37)\end{array}$ & $\begin{array}{l}(0.16- \\
0.33)\end{array}$ \\
\hline
\end{tabular}




\section{Table 4. Multivariable Linear Regression Analysis for Prehospital}

Transfer Times

\begin{tabular}{|c|c|c|c|c|c|}
\hline \multirow[t]{2}{*}{ Population density } & $0.53^{\star \star \star}$ & $0.67 * \star \star *$ & $0.26 * \star \star$ & 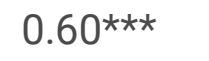 & 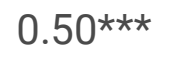 \\
\hline & $\begin{array}{l}(0.42- \\
0.64)\end{array}$ & $\begin{array}{l}(0.52- \\
0.81)\end{array}$ & $\begin{array}{l}(0.09- \\
0.44)\end{array}$ & $\begin{array}{l}(0.37- \\
0.82)\end{array}$ & $\begin{array}{l}(0.37- \\
0.63)\end{array}$ \\
\hline \multirow[t]{2}{*}{ Night time } & $1.78^{\star \star \star}$ & & & $1.15^{\star \star}$ & $2.05^{\star \star \star}$ \\
\hline & $\begin{array}{l}(1.230- \\
2.27)\end{array}$ & & & $\begin{array}{l}(0.23- \\
2.07)\end{array}$ & $\begin{array}{l}(1.48- \\
2.62)\end{array}$ \\
\hline \multirow[t]{2}{*}{ Weekend/Holiday } & 0.22 & -0.12 & $0.78^{*}$ & & \\
\hline & $\begin{array}{l}(-0.3- \\
0.74)\end{array}$ & $\begin{array}{l}(-0.77- \\
0.53)\end{array}$ & $\begin{array}{l}(-0.05- \\
1.65)\end{array}$ & & \\
\hline \multicolumn{6}{|l|}{ Severity $($ baseline $=$ mild $)$} \\
\hline \multirow[t]{2}{*}{ Moderate } & $1.87^{\star \star \star}$ & $1.97^{\star \star \star}$ & $1.68^{\star \star \star}$ & $2.69 * \star \star$ & $1.52^{\star \star \star}$ \\
\hline & $\begin{array}{l}(1.29- \\
2.46)\end{array}$ & $\begin{array}{l}(1.25- \\
2.69)\end{array}$ & $\begin{array}{l}(0.67- \\
2.69)\end{array}$ & $\begin{array}{l}(1.59- \\
3.79)\end{array}$ & $\begin{array}{l}(0.82- \\
2.21)\end{array}$ \\
\hline \multirow[t]{2}{*}{ Severe } & 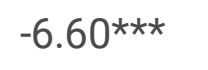 & 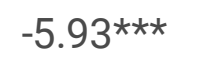 & $-7.58 * \star \star$ & $-5.83^{\star \star \star}$ & $-6.94 * \star \star$ \\
\hline & $\begin{array}{l}(-7.32- \\
-5.88)\end{array}$ & $\begin{array}{l}(-6.89- \\
-4.96)\end{array}$ & $\begin{array}{l}(-8.65- \\
-6.50)\end{array}$ & $\begin{array}{l}(-7.15- \\
-4.51)\end{array}$ & $\begin{array}{l}(-7.80- \\
-6.08)\end{array}$ \\
\hline \multirow[t]{2}{*}{ Death } & 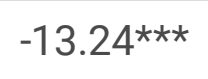 & $-13.92^{\star \star \star}$ & $-12.43^{\star \star \star}$ & $-13.03^{\star \star \star}$ & $-13.35^{\star \star \star}$ \\
\hline & $\begin{array}{l}(-13.85- \\
-12.63)\end{array}$ & $\begin{array}{l}(-14.70- \\
-13.14)\end{array}$ & $\begin{array}{l}(-13.41- \\
-11.44)\end{array}$ & $\begin{array}{l}(-14.16- \\
-11.90)\end{array}$ & $\begin{array}{l}(-14.07- \\
-12.63)\end{array}$ \\
\hline \multicolumn{6}{|l|}{ Place (baseline = home) } \\
\hline \multirow[t]{2}{*}{ Public place } & $-0.97 * \star$ & $-0.91^{\star}$ & -1.08 & -1.03 & $-0.95^{\star}$ \\
\hline & $\begin{array}{l}(-1.82- \\
-0.11)\end{array}$ & $\begin{array}{l}(-1.98- \\
0.15)\end{array}$ & $\begin{array}{l}(-2.53- \\
0.38)\end{array}$ & $\begin{array}{l}(-2.66- \\
0.60)\end{array}$ & $\begin{array}{l}(-1.96- \\
0.07)\end{array}$ \\
\hline \multirow[t]{2}{*}{ Work place } & $-2.66^{\star \star \star}$ & $-2.56^{\star \star}$ & $-2.66 * *$ & -0.92 & $-3.34 * \star \star$ \\
\hline & $\begin{array}{l}(-4.33- \\
-0.99)\end{array}$ & $\begin{array}{l}(-5.05- \\
-0.07)\end{array}$ & $\begin{array}{l}(-4.86- \\
-0.47)\end{array}$ & $\begin{array}{l}(-4.12- \\
2.28)\end{array}$ & $\begin{array}{l}(-5.29- \\
-1.40)\end{array}$ \\
\hline \multirow[t]{2}{*}{ Road } & -0.47 & -0.09 & -1.36 & -1.67 & 0.06 \\
\hline & $\begin{array}{l}(-1.83- \\
0.89)\end{array}$ & $\begin{array}{l}(-1.78- \\
1.61)\end{array}$ & $\begin{array}{l}(-3.58- \\
0.87)\end{array}$ & $\begin{array}{l}(-3.99- \\
0.64)\end{array}$ & $\begin{array}{l}(-1.61- \\
1.73)\end{array}$ \\
\hline \multirow[t]{2}{*}{ Other } & $1.94^{\star \star \star}$ & $1.62^{\star \star}$ & $2.46^{\star \star \star}$ & $2.37^{\star \star}$ & $1.74^{\star \star}$ \\
\hline & $\begin{array}{l}(0.823- \\
3.04)\end{array}$ & $\begin{array}{l}(0.22- \\
3.02)\end{array}$ & $\begin{array}{l}(0.65- \\
4.28)\end{array}$ & $\begin{array}{l}(0.39- \\
4.36)\end{array}$ & $\begin{array}{l}(0.40- \\
3.07)\end{array}$ \\
\hline Hospital beds per capita & $-0.03^{* \star *}$ & $-0.03^{\star \star *}$ & $-0.03^{\star \star *}$ & $-0.03^{\star \star \star}$ & $-0.03^{\star \star \star}$ \\
\hline
\end{tabular}




\section{Table 4. Multivariable Linear Regression Analysis for Prehospital \\ Transfer Times}

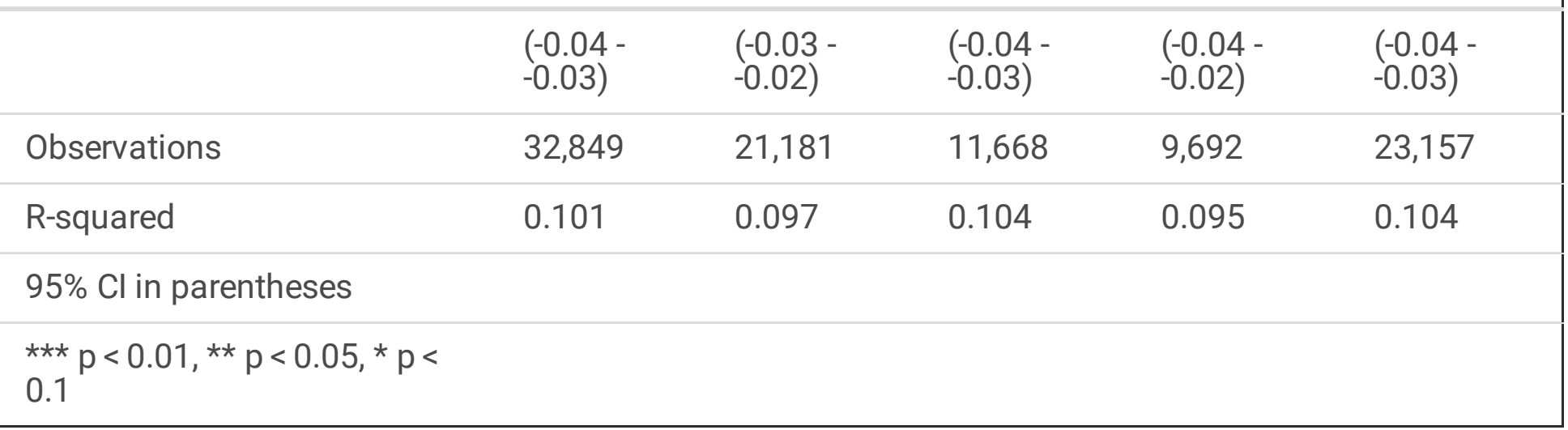

When limited to night-time transfers, EP3 and MSPH were associated less difficulty in hospital acceptance (OR $0.63,95 \% \mathrm{Cl} 0.54$ to $0.73, \mathrm{P}<0.01$, and $\mathrm{OR} 0.50,95 \% \mathrm{Cl} 0.36$ to $0.70, \mathrm{P}<0.01$, respectively) and shorter prehospital transfer time $(-4.40,95 \% \mathrm{Cl}-3.53$ to $-5.27, \mathrm{P}<0.01$, and $-2.77,95 \% \mathrm{Cl}-0.98$ to -4.56 , $\mathrm{P}<0.01$, respectively). Regarding weekend/holiday transfer, EP3 and MSPH were also associated with less difficulty in hospital acceptance (OR $0.77,95 \% \mathrm{Cl} 0.62$ to $0.95, \mathrm{P}<0.05$, and $\mathrm{OR} 0.56,95 \% \mathrm{Cl} 0.34$ to $0.94, \mathrm{P}<0.05$, respectively), and shorter prehospital transfer time $(-3.86,95 \% \mathrm{Cl}-2.58$ to $-5.13, \mathrm{P}<0.01$, and $-3.24,95 \% \mathrm{Cl}-0.86$ to $-5.62, \mathrm{P}<0.01$, respectively).

\section{Discussion}

This study analyzed large-scale nation-wide retrospective observational data recorded ambulance transfers in Japan. The data revealed that the presence of EC3s and MSPHs were associated with less difficulty in hospital acceptance and shorter prehospital transfer time. Patients with self-harm behavior have both physical and psychiatric problems that require assessment by multiple specialties including psychiatry. It can be assumed that small emergency care centers and hospitals with only psychiatry services are likely to have difficulty in treating these patients due to lack of resources, while the presence of EC3s and MSPHs are associated with less difficulty in hospital acceptance and shorter prehospital transfer time because these facilities are more likely to have the capability to treat the patients after selfharm behavior. The results of this study support the assumption.

According to White Paper on Fire Service in 2018, the number of ambulance dispatch was 1.37 times higher, and the average time required to take a patient to a hospital was 1.34 times longer, compared to $2003^{11}$. Due to the aging population, this trend is most likely to continue. Ambulance diversion and increased prehospital time are associated with higher mortality rates in certain populations, such as myocardial infarction and trauma patients ${ }^{3-5}$. Currently, there is no clear evidence that ambulance diversion and increased prehospital time are associated with worse outcomes in patients with self-harm behavior. However, increased prehospital time and ambulance diversion affect not only the patient who has difficulty finding a receiving hospital, but also other patients who need ambulances in the area. Ambulance diversion needs to be fixed systematically to maintain the EMS system to support everyone in 
need. A previous retrospective observational study revealed that self-harm is one of several factors significantly associated with difficulty in hospital acceptance ${ }^{7}$. Other studies suggested which factors are associated with difficulty in hospital acceptance in elderly and traffic accident patients ${ }^{12,13}$, but there are no known risk factors associated with difficulty in hospital acceptance in patients with self-harm behavior. Finding these factors could facilitate our understanding of the nature of the problem and a possible solution in the future. Considering the recent increase in the rate of ED visits related to suicidal attempts or suicidal ideation at all ages ${ }^{14}$, systemic solutions to decrease ambulance diversion and shorten prehospital transfer time are needed.

This study suggests that the presence of hospitals that can provide both physical and mental care is related to decreased ambulance diversion and shorter prehospital transfer time. The number of smaller emergency care centers are associated with increased difficulty in hospital acceptance and longer prehospital transfer time. The presence of hospitals with only psychiatric service are not related to difficulty in hospital acceptance and prehospital transfer time. Recently, the number of general hospitals with psychiatric inpatient services has been decreasing in Japan ${ }^{8,9}$, which might negatively affect patients with both physical and psychiatric illnesses. Based on the information obtained for the study, six EC3s without inpatient psychiatric units had opened new small-scale psychiatric inpatient units between 2013 and 2016 in an attempt to appropriately manage patients with both physical and mental problems. We need to closely monitor the effect on the EMS system with the availability of inpatient psychiatric units. Ambulance diversion might be reduced with close communication between general hospitals without psychiatric units and hospitals with only psychiatric services. Analysis by interaction terms indicated the effect of EP3 and MSPH are small when the other is present in the same city. This indicates the lack of communication and coordination between these hospitals, which is necessary to decrease the rate of difficulty in hospital acceptance and shorten pretransfer time. Our study does not offer a solution for the problem. Further studies are needed to investigate how we can create effective medical policy.

This study has some limitations. First, this is a retrospective observational study that showed the association between decreased difficulty in hospital acceptance, shorter prehospital transfer time, and the presence of EC3s/MSPHs. Therefore, we cannot conclude any causal relationship due to possible confounding factors. Second, although we have tried to include factors that might affect difficulty in hospital acceptance and prehospital transport time, such as the number of hospital beds per capita, population density, and time from dispatch to arrival at the scene, there might be unmeasured factors that were not included in the analysis. Third, the endpoints of this study are difficulty in hospital acceptance and shorter prehospital transfer time, which may not directly reflect the patient outcomes. We cannot conclude the presence of EC3s and MSPHs are associated with improved clinical outcomes. Fourth, even though we used the nation-wide database, it does not include transfers in Tokyo, the largest metropolitan area in Japan. However, Tokyo has already implemented a rule to suppress ambulance diversion and the lack of data from Tokyo might have positively affected the study. Lastly, the data used is from Japan only and the results may not be applicable to different countries. Nevertheless, we think facilities that are capable of providing both medical/surgical and psychiatric care are warranted in any 
country and could contribute to decreasing ambulance diversion for patients who need both medical/surgical and psychiatric care.

In conclusion, this retrospective observational study using nation-wide ambulance transfer data showed the association between the presence of EP3/MSPH and decreased difficulty in hospital acceptance and shorter prehospital transfer time. Further research is warranted for appropriate medical policymaking to reduce ambulance diversion and improve patient outcome for patients who need both physical and mental treatment.

\section{Declarations}

\section{Acknowledgment}

The authors are grateful to all EMS personnel who contributed to patient care and data collection. We also would like to thank Japanese Society of General Hospital Psychiatry who provided the information about general hospitals with psychiatric inpatient units.

\section{Author Contributions}

T.C., R.T., H.T., S.I. and T.S. contributed to the study concept and design. R.T. and E.I. performed data curation and formal analysis. T.C. wrote the initial draft of the manuscript. M.R., H.T., S.I. and T.S. contributed to critical review and preparation of the manuscript. T.C. and T.S. supervised and lead the project. All authors reviewed and approved the final version of the manuscript.

\section{Competing interests}

The authors declare no competing interests.

\section{References}

1. Redelmeier, D. A., Blair, P. J. \& Collins, W. E. No place to unload: A preliminary analysis of the prevalence, risk factors, and consequences of ambulance diversion. Ann. Emerg. Med. 23, 43-47 (1994).

2. Katayama, Y. et al. Factors associated with the difficulty in hospital acceptance at the scene by emergency medical service personnel: a population-based study in Osaka City, Japan. BMJ Open, 6, e013849 (2016).

3. Hatta, K., Nakamura, H., Usui, C. \& Kurosawa, H. Utility and sufficiency of psychiatric inpatient units in general hospitals: A cross-sectional study in Tokyo. Psychiatry Clin. Neurosci, 64, 642-644 (2010).

4. Committee, M. T. P. J. S. of G. H. P. Current Status of Japanese General Hospital Psychiatry in 2008. Jpn. J. Gen. Hosp. Psychiatry, 22, 55-64 (2010).

5. Higashi, H., Takaku, R., Yamaoka, A., Lefor, A. K. \& Shiga, T. The Dedicated Emergency Physician Model of emergency care is associated with reduced pre-hospital transportation time: A retrospective 
study with a nationwide database in Japan. PLOS ONE, 14, e0215231 (2019).

6. Fire and Disaster Management Agency. 2018 White Paper on Fire Service https://www.fdma.go.jp/publication/hakusho/h30/46816.html. (2019)

7. Matsuyama, T. et al. Factors associated with the difficulty in hospital acceptance among elderly emergency patients: A population-based study in Osaka City, Japan: Hospital acceptance difficulty. Geriatr. Gerontol. Int, 17, 2441-2448 (2017).

8. Katayama, Y. et al. Evaluation of factors associated with the difficulty in finding receiving hospitals for traffic accident patients at the scene treated by emergency medical services: a population-based study in Osaka City, Japan. Acute Med. Surg, 4, 401-407 (2017).

9. Owens, P. L., McDermott, K. W., Lipari, R. N. \& Hambrick, M. M. Emergency Department Visits Related to Suicidal Ideation or Suicide Attempt, 2008-2017: Statistical Brief \#263. in Healthcare Cost and Utilization Project (HCUP) Statistical Briefs(Agency for Healthcare Research and Quality (US), 2006).

10. 10. Higashi, H., Takaku, R., Yamaoka, A., Lefor, A. K. \& Shiga, T. The Dedicated Emergency Physician Model of emergency care is associated with reduced pre-hospital transportation time: A retrospective study with a nationwide database in Japan. PLOS ONE 14, e0215231 (2019).

11. 11. Fire and Disaster Management Agency. 2018 White Paper on Fire Service https://www.fdma.go.jp/publication/hakusho/h30/46816.html. (2019)

12. 12. Matsuyama, T. et al. Factors associated with the difficulty in hospital acceptance among elderly emergency patients: A population-based study in Osaka City, Japan: Hospital acceptance difficulty. Geriatr. Gerontol. Int. 17, 2441-2448 (2017).

13. 13. Katayama, Y. et al. Evaluation of factors associated with the difficulty in finding receiving hospitals for traffic accident patients at the scene treated by emergency medical services: a population-based study in Osaka City, Japan. Acute Med. Surg. 4, 401-407 (2017).

14. 14. Owens, P. L., McDermott, K. W., Lipari, R. N. \& Hambrick, M. M. Emergency Department Visits Related to Suicidal Ideation or Suicide Attempt, 2008-2017: Statistical Brief \#263. in Healthcare Cost and Utilization Project (HCUP) Statistical Briefs (Agency for Healthcare Research and Quality (US), 2006).

\section{Figures}




\section{Background}

Ambulance transfer after self-harm behavior is associated with difficulty in hospital acceptance and longer prehospital transfer time
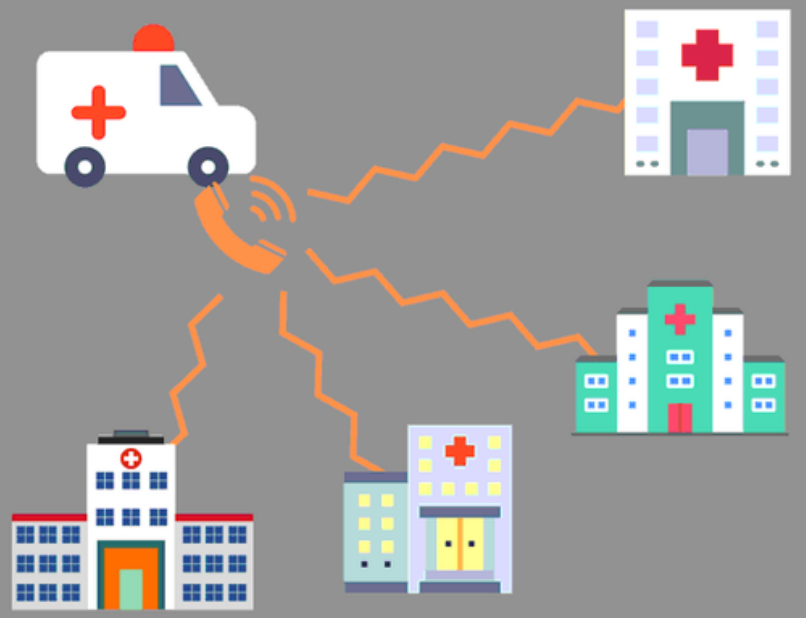

\section{Method}

A retrospective observational study using a Japanese national database of nationwide ambulance dispatches in 2015.

\section{Result}

Difficulty in Hospital Acceptance

(Phone calls $\geq 4$ )

Prehospital transfer time

$8.4 \%(2767 / 32849)$

\section{1 min}

\section{High-Level Emergency Care Centers} (Level Three Emergency Care Centers) Difficulty in Hospital Acceptance

$$
\begin{gathered}
\text { OR }=0.63 \\
(0.55-0.71) \\
\text { Prehospital transfer time } \\
\text { (b) }-4.21 \text { min } \\
(-4.89 \text { to }-3.53)
\end{gathered}
$$

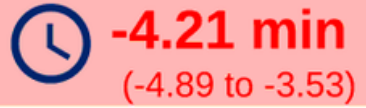

Hospitals with Both Medical/Surgical and Psychiatric Inpatient Beds

Difficulty in Hospital Acceptance

$$
\text { ( } \mathrm{OR}_{(0.38-0.66)}^{0.50}
$$

Prehospital transfer time

(C) -3.46 min

Conclusion High-Level Emergency Care Centers and Hospitals with Both Medical/Surgical and Psychiatric Inpatient Beds are associated with significant decrease in difficulty in hospital acceptance and shorter prehospital transfer time.

Figure 1

The graphical summary of this study. 


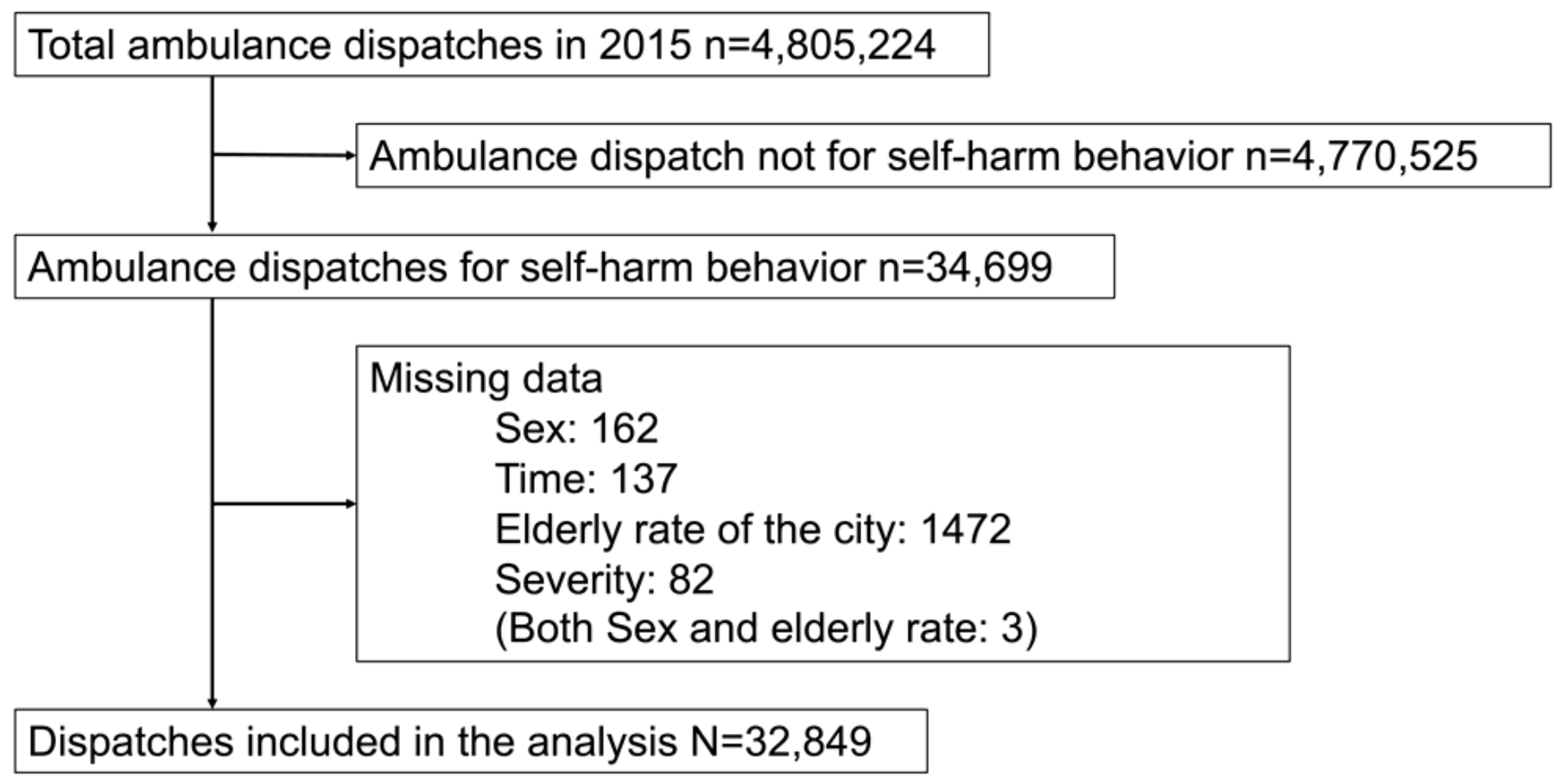

Figure 2

Flow charts of the patients included in the analysis.

\section{Supplementary Files}

This is a list of supplementary files associated with this preprint. Click to download.

- Tables.docx 